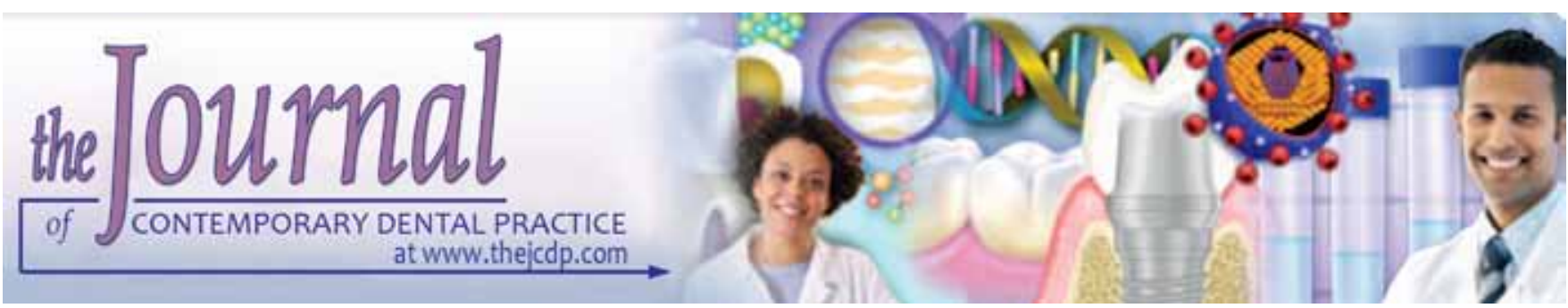

\title{
Antibiofilm and Antimicrobial Activity of Polyethylenimine: An Interesting Compound for Endodontic Treatment
}

\author{
1Joana Barros, ${ }^{2}$ Ana Dias, ${ }^{3}$ Miguel A Rodrigues, ${ }^{4}$ Cidália Pina-Vaz, ${ }^{5}$ Maria A Lopes, ${ }^{6}$ Irene Pina-Vaz
}

\begin{abstract}
Aim: Bacteria levels of necrotic teeth are greatly reduced after endodontic treatment procedures but the presence of persisting microorganisms leads to continuous efforts to develop materials with antimicrobial properties. The purpose of the study was to determine the antimicrobial activity of polyethylenimine (PEI) against common bacteria and yeasts, regarding planktonic cells and biofilm, and to clarify its antimicrobial mechanism of action through flow cytometry.
\end{abstract}

Materials and methods: The antibiofilm and antimicrobial effect of PEI was determined against Enterococcus faecalis, Staphylococcus aureus, Escherichia coli and Candida albicans strains using reference protocols. The effect of PEI was evaluated regarding adhesion, biofilm formation and biofilm disaggregation. In order to understand PEI cellular effects flow cytometric analysis was performed with different fluorescent markers.

Results: It was verified that minimal inhibitory concentrations (MIC) values and minimal lethal concentrations (MLC) obtained for PEI were similar and ranged between 50 and $400 \mathrm{mg} / \mathrm{l}$, proving the microbicidal and fungicidal activity of this compound. Antibiofilm activity was also proved for all the microorganisms. Severe lesion of the membrane and cell depolarization was demonstrated.

\footnotetext{
${ }^{1,6}$ Department of Endodontics, Faculty of Dental Medicine University of Porto, Porto, Portugal

${ }^{2,4}$ Department of Microbiology, Faculty of Medicine, University of Porto, Porto; Cardiovascular Research and Development Unit, Faculty of Medicine, University of Porto, Porto, Portugal

${ }^{3,5}$ Department of Metallurgical and Materials Engineering Faculty of Engineering, University of Porto, Porto; Centre for Mechanical Engineering of the University of Coimbra Portugal
}

Corresponding Author: Joana Barros, Professor, Department of Endodontics, Faculty of Dental Medicine, University of Porto Porto, Portugal, Phone: +351 220901 100, e-mail: joana. barros.c@gmail.com
Conclusion: Polyethylenimine showed antimicrobial and antibiofilm activity against microorganisms often associated with apical periodontitis.

Clinical significance: Theoretically, prolonging the antibacterial effects of materials used in endodontics may be interesting to help prevent reinfection and possibly to affect residual bacteria that survived the treatment procedures.

Keywords: Antimicrobial activity, Biofilms, Endodontic treatment, Polyethylenimine.

How to cite this article: Barros $\mathrm{J}$, Dias A, Rodrigues MA, Pina-Vaz C, Lopes MA, Pina-Vaz I. Antibiofilm and Antimicrobial Activity of Polyethylenimine: An Interesting Compound for Endodontic Treatment. J Contemp Dent Pract 2015;16(6):427-432

\section{Source of support: Nil}

\section{Conflict of interest: None}

\section{INTRODUCTION}

Endodontic infections are polymicrobial and although the root canal preparation, which is essentially a chemomechanical procedure, can clearly reduce microbial levels, detectable levels of bacteria are still observed in a considerable number of situations. ${ }^{1}$ Endodontic treatment failure is frequently associated with Grampositive bacteria and fungi, Enterococcus faecalis and Candida albicans are frequently detected. ${ }^{2,3}$ Consequently, numerous investigations have assessed the antimicrobial effects of irrigants, intracanal dressings and root canal sealers against these species from root canals of teeth with post-treatment apical periodontitis. ${ }^{4,5}$ Currently, endodontic infections have been recognized as biofilmsinduced disease. ${ }^{6,7}$ In order to successfully treat these persistent biofilm-mediated infections, new materials and protocols have been developed additionally to standard endodontic antimicrobial procedures, including application of ozone, laser technology, and photodynamic therapy (PDT). ${ }^{8-10}$ 
Polyethylenimine (PEI) is a weekly basic, aliphatic, nontoxic synthetic polymer which is polycationic as a result of the presence of primary, secondary, and tertiary amino groups. ${ }^{11}$ The PEI polymer is used for many purposes, such as in gene delivery therapy, in the development of catalyst supports due to effective neutralization of excess anionic colloidal charge, especially under acidic and neutral $\mathrm{pH}$ conditions and as a common ingredient involved in microbicidal compositions in a variety of formulations ranging from washing agents to packaging materials. ${ }^{11-14}$ It is well known that certain polycationic substances can increase the permeability of Gram-negative bacteria outer membrane (OM) to solute that were usually unable to penetrate it, allowing a better antibacterial effect. The prevention of biofilm formation of C. albicans as well as future development and application in medical devices, has been reported. ${ }^{15}$ Its antimicrobial and antibiofilm activity against endodontic microorganisms involved in persistent infections has not been assessed yet.

The purpose of this study was to determine the antimicrobial activity of PEI against microbial strains usually involved in persistent endodontic infections in the planktonic and specially in biofilm form, and to clarify the cellular mechanism of action through flow cytometry.

\section{MATERIALS AND METHODS}

\section{Reagents}

Mueller-Hinton Broth (MHB), Mueller Hinton Agar (MHA), Brain Heart Infusion Broth (BHIB), Brain Heart Infusion Agar (BHIA), Sabouraud Dextrose Broth and Sabouraud Dextrose Agar from Liofilchem were prepared accordingly to manufacturer instructions. Phosphate buffered saline solution pH 7.4 (PBS) and RPMI 1640 medium supplemented with L-glutamine and buffered with MOPS acid, both from Sigma-Aldrich, were prepared accordingly to manufacturer instructions. Polyethylenimine (branched poly (ethyleneimine) solution, average $\mathrm{Mw} \sim 750.000$, reference 181978; SigmaAldrich) stock solution at $25 \%(\mathrm{w} / \mathrm{v})$ was used in the experiments.

\section{Microbial Strains}

A strain from American type culture collection (ATCC) and a clinical isolate of each microorganism were used: E. faecalis (ATCC 29212 and EF1), Staphylococcus aureus (ATCC 25923 and SA1), Escherichia coli (ATCC 25922 and CO1) and C. albicans (ATCC 90028 and CA1). Clinical isolates were identified by Vitek2 system (bioMérieux,
Vercieux, France). Bacterial strains were kept frozen in Luria-Bertani broth (LB) (Difco Laboratories, Detroit, MI, USA) supplemented with $20 \%$ glycerol and yeast strains in yeast peptone dextrose medium (YPD) (Difco Laboratories, Detroit, MI, USA) supplemented with $40 \%$ glycerol at $-70^{\circ} \mathrm{C}$ until testing. For each experiment, the microorganisms were subcultured twice on LB agar at $37^{\circ} \mathrm{C}$ for 24 hours (bacteria) or Sabouraud agar at $35^{\circ} \mathrm{C}$ for 24 hours (yeasts) to assess the purity of the culture and its viability.

\section{Antimicrobial Susceptibility Assay}

Minimal inhibitory concentrations (MIC) of PEI were determined according to the CLSI microdilution reference protocol M100-S23, 2013 for bacteria and protocol M27-A3, 2012 for yeasts. The tested concentrations ranged from 3200 to $6.25 \mathrm{mg} / \mathrm{l}$ of PEI. The MIC endpoint was defined as the lowest drug concentration that completely inhibited the growth of microorganisms in microdilution wells. To determine the minimal lethal concentration (MLC), $20 \mu \mathrm{L}$ of each microdilution well was plated in MHA (for bacteria) or Sabouraud agar (for yeasts). The plates were incubated at $37^{\circ} \mathrm{C}$ for 24 hours (bacterial strains) or $35^{\circ} \mathrm{C}$ for 48 hours (yeast strains) with subsequent CFU counting. The MLC was defined as the lowest drug concentration that killed at least $99.9 \%$ of the final inoculum.

\section{Adhesion and Biofilm Assays}

For all assays, bacterial and yeast cells were grown overnight at $37^{\circ} \mathrm{C}, 180 \mathrm{RPM}$ in MHB (bacteria) or Sabouraud (yeasts) broth. After incubation, cells were harvested by centrifugation (5000 gm, 5 minutes), washed with phosphate-buffered saline (PBS) and the inoculum was adjusted with a hemacytometer to a standardized suspension of $1 \times 10^{7} \mathrm{cell} / \mathrm{ml}$ in MHB for bacteria and $1 \times 10^{6}$ cells $/ \mathrm{ml}$ in RPMI for yeast.

1. To evaluate the effect of PEI in the microorganisms adhesion to polystyrene, 1 milliliter of yeast cells suspension was placed in a 12 well polystyrene plate coated with PEI in three concentrations 200, 400 and $800 \mathrm{mg} / 1$. Subsequently, plates were incubated for 90 minutes at $37^{\circ} \mathrm{C}, 150 \mathrm{RPM}$. After adhesion time, wells were washed with PBS and adhesion was quantified by the semiquantitative 2,3-bis-(2-methoxy-4-nitro5-sulfophenyl)-5-[(phenylamino) carbonyl]-2Htetrazolium hydroxide (XTT) reduction assay.

2. In order to evaluate if PEI has the ability to impair biofilm formation, biofilms of the different species were allowed to grow in polystyrene plates for 24 hours in the presence of PEI. The same concentrations of PEI 
were used (200, 400 and $800 \mathrm{mg} / \mathrm{l})$. After incubation PEI effect was measured spectrophotometrically by quantifying biofilm metabolic activity, using the XTT reduction assay.

3. In order to study biofilm disaggregation yeast and bacterial biofilms were formed in polystyrene 12 well plates, for 24 hours at $37^{\circ} \mathrm{C}$. After that, biofilms were washed twice with PBS and challenged with PEI, in the concentrations previously used, for additional 24 hours. The effect of PEI in biofilm disaggregation was evaluated with XTT reduction assay.

\section{Antimicrobial Mechanism of Action}

After incubation for 60 minutes with PEI, the different microorganisms were stained with two fluorescent markers: Propidium iodide (PI) (Molecular Probes Europe BV, Leiden, Netherlands), a death marker which binds to DNA of cells with cytoplasmatic membrane severally damaged, and bis-(1,3-dibutylbarbituric acid) trimethine oxonol (DIBAC $\left.{ }_{4}(3)\right)$ (Molecular probes) which is a lipophilic and anionic fluorescent stain which is accumulated intracellularly when the cytoplasmatic membrane is depolarized. Additionally, FUN-1 a fluorescent probe that measures yeast metabolic activity was used. Flow cytometric analysis was performed with cellQuest TM pro Software and was based on light-scatter and fluorescence signals resulting from $15 \mathrm{~mW}$ laser illumination at $488 \mathrm{~nm}$ and $635 \mathrm{~nm}$. Signals corresponding to forward and side scatter (FSC and SSC) and fluorescence were accumulated at FLI (530/30 nm) for $\mathrm{DIBAC}_{4}(3), \mathrm{FL} 2(620 \mathrm{~nm})$ for FUN-1 and FL3 $(670 \mathrm{~nm})$ for PI. For sample preparation, $1 \times 10^{6}$ cells $/ \mathrm{ml}$ were treated with MLC of PEI for 15 to 90 minutes. After treatment with PEI, the different strains were incubated with each fluorescent probe for 30 minutes in the dark at $1 \mu \mathrm{g} / \mathrm{ml}$ for $\mathrm{DIBAC}_{4}(3)$ and PI and $0.5 \mu \mathrm{M}$ for FUN-1. Control suspensions, not exposed to any treatment were used as auto fluorescence. Cells not treated with PEI and stained with fluorescent probes were used as viable control. At least 30,000 cells from each suspension were analyzed on a BD Biosciences FACS Calibur.

\section{Data Analysis and Statistic Treatment}

Biofilm metabolic activity was expressed as the percentage in relation to the control in the presence of PEI. Adhesion and biofilm inhibitory effect, in different concentrations, was evaluated with one-way ANOVA with the Bonferroni correction. The student's test was used to evaluate the activity of treatment in pre-formed biofilms. Statistical significance was considered as a p-value inferior to 0.05. All statistical analyses were conducted using the SPSS software (v. 20.0).

\section{RESULTS}

\section{Antimicrobial Activity}

Polyethylenimine MIC for bacteria was $400 \mathrm{mg} / 1$ and for yeast was $50 \mathrm{mg} / 1$. The MLC values obtained for PEI were equal to the MIC values. The values obtained for clinical and ATCC strains were similar.

\section{Adhesion and Biofilm Formation}

All strains adhered promptly to polystyrene plates, however, this adherence was significantly reduced in the presence of all tested concentrations of PEI (Fig. 1). Polyethylenimine significantly inhibited the biofilm formation and, probably more important in vivo, it was able to disaggregate it in great extension regarding all microorganisms even in the lower concentrations.

\section{Antimicrobial Mechanism of Action}

Severe lesion of the membrane due to PEI exposition (MLC values) for a short period of time was demonstrate as around $50 \%$ of the cells were PI positive (Figs 2C and D) and depolarization of the cells was shown by the increase of staining of the cells after staining with $\operatorname{DIBAC}_{4}(3)$ on bacteria (Figs 2A and B). Similar results were obtained with yeasts, as well as metabolic disturbance, shown by an increase of FUN-1 staining.

\section{DISCUSSION}

The relevant antibiofilm effect and wide spectrum activity of PEI, both against Gram-positive and Gram-negative bacteria as well as against yeasts turn it a really promising compound for incorporation or coating in endodontic materials. Regarding C. albicans a marked reduction in the biofilm formation was already described when PEI coated the modified surfaces of a polymer, frequently used in the production of indwelling devices, such as catheters and voice prostheses. ${ }^{16}$ In the present study adhesion, the first step for infection was significantly reduced in the presence of PEI. Moreover, a dosedependent inhibition of biofilm formation and biofilm disaggregation was also verified. In what concerns to bacteria these events occurred even in the presence of subinhibitory concentrations of PEI. This fact is particularly interesting in the case of biofilm disaggregation once it is well known that cells organized in the biofilm lifestyle have increased tolerance to the classical antimicrobial drugs. The tested concentrations were chosen in order to include the bacteria MIC/MLC, $2 \times \mathrm{MIC}$ and a subinhibitory concentration ( $1 / 2 \mathrm{MIC})$. This option was made for three reasons: bacteria are more frequent in endodontic infection than yeasts; bacteria were much 


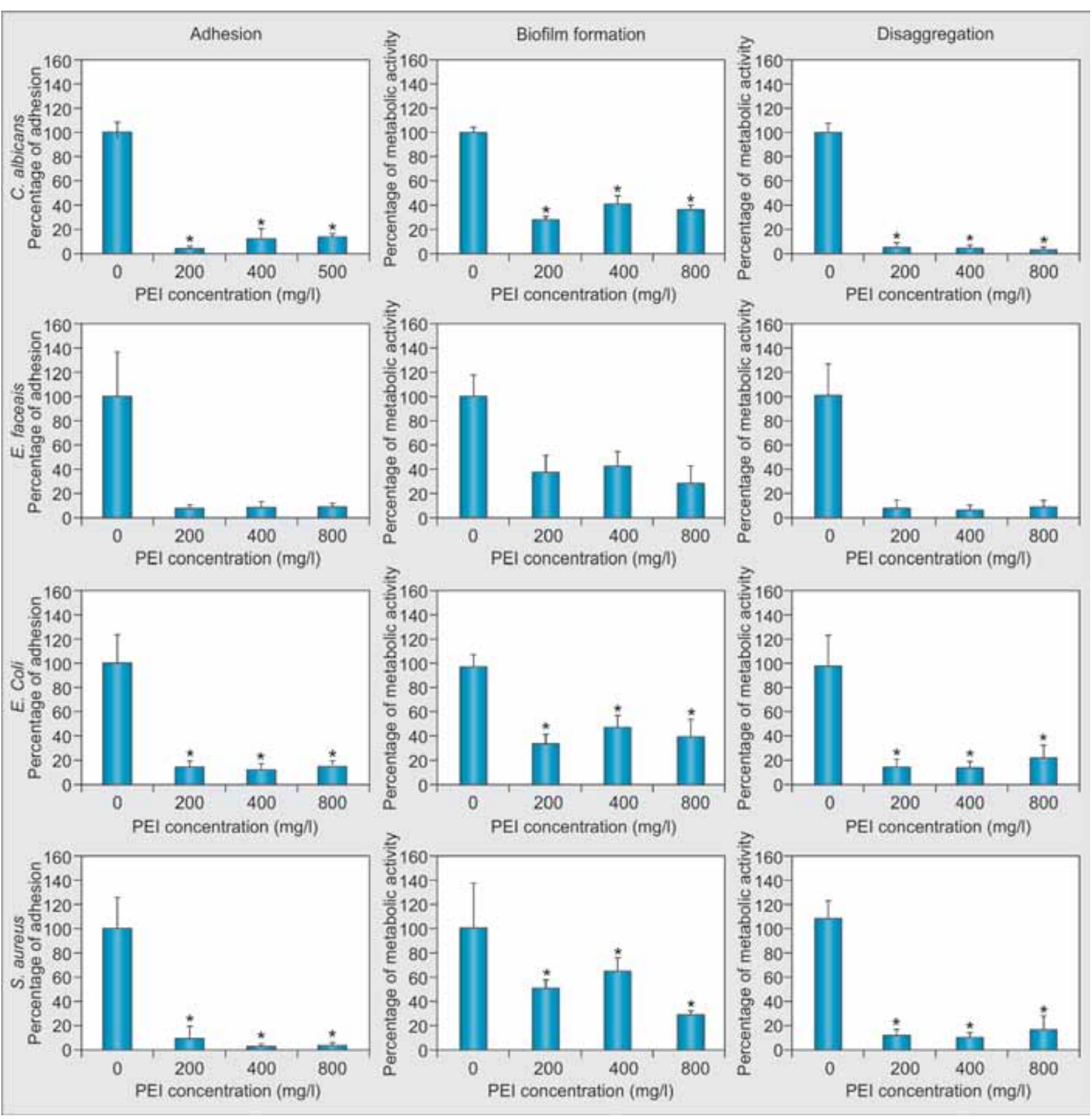

Fig. 1: Effect of PEI on adhesion, biofilm formation and biofilm disaggregation measured as percentage of cells metabolically active (XTT method) developed on polystyrene plates regarding the ATCC tested microorganisms

more resistant than yeasts to PEI and finally because our goal was to elect the better PEI concentration that inhibit all microorganisms, for a possible incorporation or coating of endodontic materials.

According with the antimicrobial susceptibility assay, PEI showed a bactericidal effect for both Gram-negative and Gram-positive bacteria and even more active effect as fungicidal (MIC value equal to MLC value). This broad spectrum is rather important as endodontic infections could have different etiologies and are often polymicrobial. By other side, it indicates that the target of PEI should be common to all those microorganisms.
Flow cytometry have been proved as an excellent tool in microbiology field to quickly demonstrate antimicrobial activity as well as to clarify about mechanism of action/resistance. ${ }^{17,18}$ Polyethylenimine staining showed the ability of PEI in producing severe lesion of the cytoplasmic membrane in bacteria and in yeasts after short incubation time. Furthermore, it depolarizes cell membrane in bacteria and disturbs the yeast metabolism. A study reported that, in contrast to other permeabilizers, PEI does not induce the release of lipopolysaccharide (LPS) from the outer membrane (OM) but rather a change in the distribution of phospholipids from the inner to the 

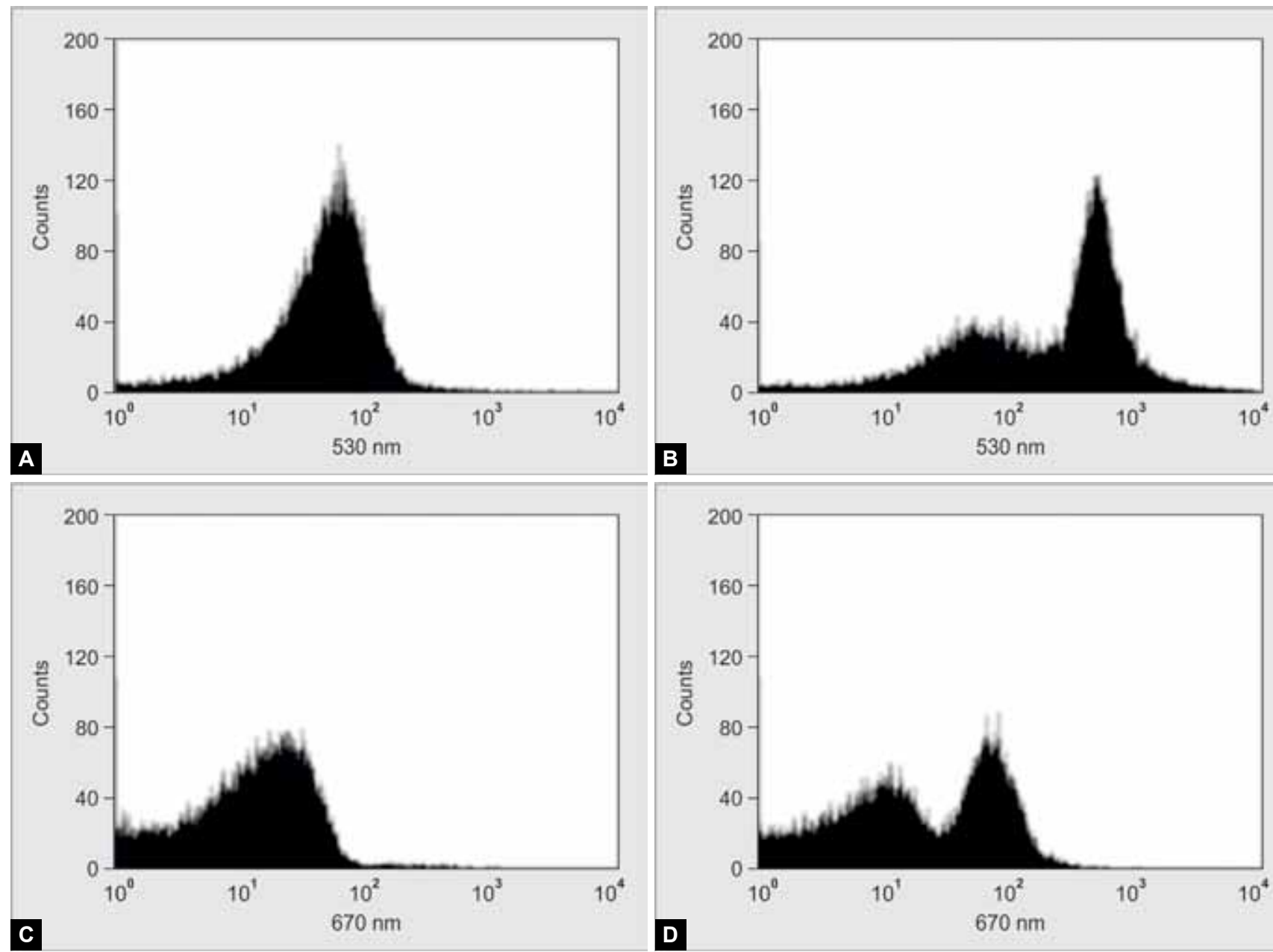

B

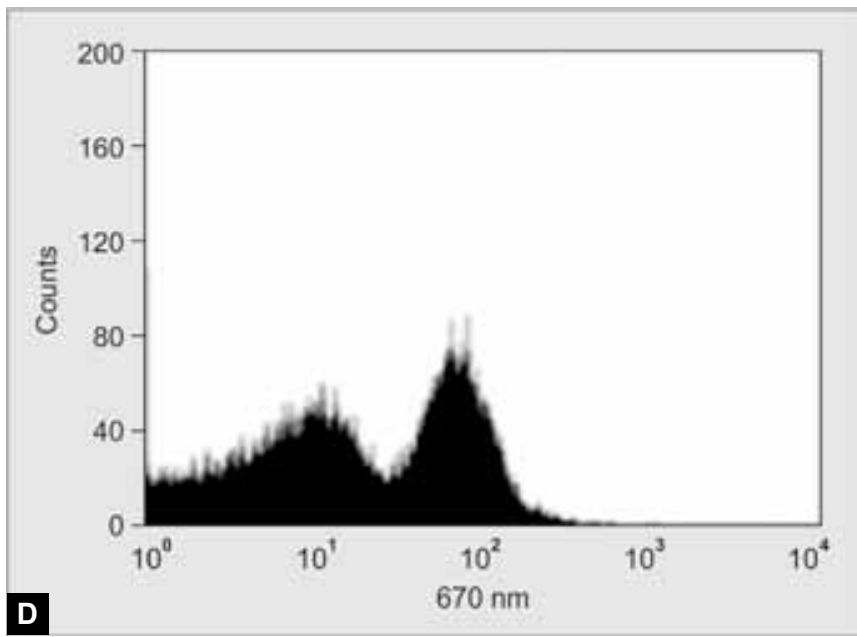

Figs 2A to D: Representative histograms of flow cytometry analysis regarding Enterococcus faecalis cells not treated (A and C) and treated with MLC values of PEI (B and $D)$, stained with $\operatorname{DIBAC}_{4}(3)(A$ and $B)$ and $\mathrm{PI}(C$ and $D)$. The intensity of fluorescence was measured at FL1 $(530 / 30 \mathrm{~nm})$ and at FL3 $(670 \mathrm{~nm})$.

outer layer, that increase the permeability. ${ }^{19}$ A reduction of the bactericidal activity of some antibiotics by PEI, particularly hydrophilic positively charged, may be due to a competition for the same binding. Polyethylenimine having higher positive charge when compared with other compounds may be the cause of the greater affinity between it and OM, greater than the ones between antibiotics and $\mathrm{OM}^{20}$

Unlike classical antimicrobial agents, whose activity is due to the leaching of antimicrobial agents at subinhibitory concentrations, leading to emergence of resistance, the antimicrobial polymers have the advantage of achieving a long antimicrobial activity without releasing toxic products. ${ }^{21}$ The mechanism of action through lesion of membrane may explain the cytotoxicity of PEI. ${ }^{22}$ This only occurs in the contact area which can have different implications depending on the biomedical application. In the case of endodontic sealers, coating the surface of the instruments (NiTi) or gutta-percha cones the tissue contact is almost none and the increasing of long-term antimicrobial activity is advantageous. The synthesis of nanoparticles of PEI (QPEI nanoparticles) with antibacterial properties has been described. ${ }^{23}$ Unlike the solubility and hydrophilic's characteristics of PEI, these QPEI nanoparticles were referred as insoluble and hydrophobic, whose mechanism of action is due to direct contact only. Furthermore, different from the common antimicrobial agents, there is no leakage of toxic compounds and its higher positive charge means a stronger antibacterial effect against oral bacteria. Due to high surface area, their effect is mediated through a small load added to the materials. These properties should ensure the stability of materials with long-term antimicrobial activity, ideally without compromising their physiochemical and mechanical properties.

\section{CONCLUSION}

The discoveries of this study confirmed the antimicrobial activity of PEI against microorganisms often associated with apical periodontitis refractory to endodontic treatment. In general, in order to increase the antimicrobial activity of biomedical devices, further research is being conducted to evaluate the inclusion of PEI compound which may play a promising role in future. Therefore, 
especially QPEI nanoparticles, seems to have the potential to improve the antibacterial effect of endodontic materials such sealers, either by increasing efficacy in the fresh state or generating prolonged effects, or both.

\section{CLINICAL SIGNIFICANCES}

Despite the existence of several antimicrobial agents none seems to be efficient for the elimination of nutrients, as necrotic pulp tissue and microorganisms, during endodontic treatment. Due to effectiveness of PEI can be considered as an interesting compound for endodontic treatment which can emphasize its possible role by incorporating or coating materials in order to achieve a better outcome.

\section{ACKNOWLEDGMENTS}

The authors thank Patricia Ramalho for technical assistance. The authors would like to acknowledg the financial support from FEDER funds through the program COMPETE-Programa Operacional Factores de Competitividade- under the project PEst-C/EME/ UI0285/2011.

\section{REFERENCES}

1. Rôças IN, Siqueira JF Jr. In vivo antimicrobial effects of endodontic treatment procedures as assessed by molecular microbiologic techniques. J Endod 2011;37:304-310.

2. Siqueira JF Jr, Rôças IN. Clinical implications and microbiology of bacterial persistence after treatment procedures. J Endod 2008;34:1291-1301.

3. Blome B, Braun A, Sobarzo V, Jepsen S. Molecular identification and quantification of bacteria from endodontic infections using real-time polymerase chain reaction. Oral Microbiol Immunol 2008;23:384-390.

4. Malkhassian G, Manzur AJ, Legner M, Fillery ED, Manek S, Basrani BR, Friedman S. Antibacterial efficacy of MTAD final rinse and two percent chlorhexidine gel medication in teeth with apical periodontitis: a randomized double-blinded clinical trial. J Endod 2009;35:1483-1490.

5. Stuart CH, Schwartz SA, Beeson TJ, Owatz CB. Enterococcus faecalis: its role in root canal treatment failure and current concepts in retreatment. J Endod 2006;32:93-98.

6. Ricucci D, Siqueira JF Jr. Biofilms and apical periodontitis: study of prevalence and association with clinical and histopathologic findings. J Endod 2010;36:1277-1288.

7. Svensäter GBG. Biofilms in endodontic infections. Endodontic Topics 2004;9:27-36.

8. Hems RS, Gulabivala K, Ng YL, Ready D, Spratt DA. An in vitro evaluation of the ability of ozone to kill a strain of Enterococcus faecalis. Int Endod J 2005;38:22-29.

9. Martins MR, Carvalho MF, Vaz IP, Capelas JA, Martins MA, Gutknecht N. Efficacy of Er, Cr: YSGG laser with endodontical radial firing tips on the outcome of endodontic treatment: blind randomized controlled clinical trial with 6-month evaluation. Lasers Med Sci 2012: DOI:10.1007/s10103-012-1172-1176.

10. Rios A, He J, Glickman GN, Spears R, Schneiderman ED, Honeyman AL. Evaluation of photodynamic therapy using a light-emitting diode lamp against Enterococcus faecalis in extracted human teeth. J Endod 2011;37:856-859.

11. Helander IM, Alakomi HL, Latva-Kala K, Koski P. Polyethyleneimine is an effective permeabilizer of gram-negative bacteria. Microbiology 1997;143:3193-3199.

12. Weber ND, Merkel OM, Kissel T, Munoz-Fernandez MA. PEGylated poly(ethylene imine) copolymer-delivered siRNA inhibits HIV replication in vitro. J Control Release 2012;157: 55-63.

13. Breunig M, Hozsa C, Lungwitz U, Watanabe K, Umeda I, Kato $\mathrm{H}$, Goepferich A. Mechanistic investigation of poly(ethylene imine)-based siRNA delivery: disulfide bonds boost intracellular release of the cargo. J Control Release 2008;130:57-63.

14. Bellettini IC, Nandi LG, Eising R, Domingos JB, Machado VG, Minatti E. Properties of aqueous solutions of hydrophobically modified polyethylene imines in the absence and presence of sodium dodecylsulfate. J Colloid Interface Sci 2012;370:94-101.

15. Honraet K, Goetghebeur E, Nelis HJ. Comparison of three assays for the quantification of Candida biomass in suspension and CDC reactor grown biofilms. J Microbiol Methods 2005; 63:287-289.

16. De Prijck K, De Smet N, Coenye T, Schacht E, Nelis HJ. Prevention of Candida albicans biofilm formation by covalently bound dimethylaminoethylmethacrylate and polyethylenimine. Mycopathologia 2010;170:213-221.

17. Pina-vaz C, Rodrigues AG, Costa-de-Oliveira S, Ricardo E, Mardh PA. Potent synergic effect between ibuprofen and azoles on Candida resulting from blockade of efflux pumps as determined by FUN-1 staining and flow cytometry. J Antimicrob Chemother 2005;56:678-685.

18. Pina-Vaz C, Rodrigues AG. Evaluation of antifungal susceptibility using flow cytometry. Methods Mol Biol 2010;638: 281-289.

19. Helander IM, Latva-Kala K, Lounatmaa K. Permeabilizing action of polyethyleneimine on Salmonella typhimurium involves disruption of the outer membrane and interactions with lipopolysaccharide. Microbiology 1998;144:385-390.

20. Khalil H, Chen T, Riffon R, Wang R, Wang Z. Synergy between polyethylenimine and different families of antibiotics against a resistant clinical isolate of Pseudomonas aeruginosa. Antimicrob Agents Chemother 2008;52:1635-1641.

21. Gould IM. A review of the role of antibiotic policies in the control of antibiotic resistance. J Antimicrob Chemother 1999; 43:459-465.

22. Brunot C, Ponsonnet L, Lagneau C, Farge P, Picart C, Grosgogeat $\mathrm{B}$. Cytotoxicity of polyethyleneimine (PEI), precursor base layer of polyelectrolyte multilayer films. Biomaterials 2007;28:632-640.

23. Beyth N, Yudovin-Farber I, Bahir R, Domb AJ, Weiss EI. Antibacterial activity of dental composites containing quaternary ammonium polyethylenimine nanoparticles against Streptococcus mutans. Biomaterials 2006;27:3995-4002. 